\title{
COMPORTAMIENTO DE LAS REDES DE BAJA TENSIÓN ANTE LA PRESENCIA DE GENERACIÓN FOTOVOLTAICA
}

\section{LOW-VOLTAGE NETWORKS' BEHAVIOUR UNDER THE PRESENCE OF PHOTOVOLTAIC GENERATION}

Joel Villavicencio-Gastelu ${ }^{1 *}{ }^{*}$, Joel Melo-Trujillo ${ }^{2}(\mathbb{0}$

Antônio Padilha-Feltrin ${ }^{1 /}$

${ }^{1}$ Sao Paulo State University, Dept. of Electrical Engineering- UNESP, Ilha Solteira, Brazil

${ }^{2}$ Federal University of ABC - UFABC, The Engineering, Modeling and Applied Social Sciences Center - CECS, Santo André, Brazil

Recibido (Recieved): 28/11/2019 Aceptado (Accepted): 04/04/2021

\section{RESUMEN}

El impacto de la generación fotovoltaica en el comportamiento de la red eléctrica depende de la cantidad de potencia inyectada. Así, durante el análisis de la red, todos los factores que pueden influenciar esa potencia deben ser considerados. En este trabajo, una metodología para analizar el comportamiento de la red ante la presencia de generación fotovoltaica es presentada. La metodología propuesta tiene en cuenta la naturaleza estocástica de la inserción fotovoltaica en la red a través del método de simulación de Monte Carlo. A fin de evaluar la influencia de la coincidencia entre la generación fotovoltaica y la demanda, una red de baja tensión trifásica con usuarios residenciales y comerciales es considerada. Las simulaciones son realizadas a lo largo de un día y considerando varios niveles de penetración fotovoltaica. Por lo tanto, la metodología presentada puede ser utilizada en los estudios de planeamiento de la operación realizados por las concesionarias de energía.

Palabras Clave: Pérdida de energía activa, redes eléctricas de baja tensión, sistemas fotovoltaicos, sobretensión

\section{ABSTRACT}

The impact of photovoltaic generation in the electrical network' behaviour depends on the quantity of injected power. Thus, during the network analysis, all factor that could influence that power must be considered. In this paper, a methodology to analyse the network' behaviour under the presence of photovoltaic generation is presented. The proposed methodology takes into account the stochastic nature of the photovoltaic insertion in the network via the Monte Carlo simulation method. In order to evaluate the influence of coincidence between PV generation and demand, a three-phase low-voltage network with residential and commercial users is considered. Simulations are performed along a day and considering several levels of photovoltaic penetration. Therefore, the proposed methodology can be used in operation planning studies performed by utilities.

Keywords: Active energy loss, low-voltage networks, photovoltaic system, overvoltage

\section{INTRODUCCIÓN}

En los últimos años, la instalación de fuentes renovables de energía a aumentado alrededor del mundo [1]. Entre esas fuentes renovables se encuentra la energía solar, la cual puede ser convertida en energía eléctrica a través de los sistemas fotovoltaicos.

La conexión de los sistemas fotovoltaicos en la red eléctrica modifica los flujos de potencia en las líneas. En general, la presencia de los sistemas fotovoltaicos ocasiona una elevación de la tensión en la red. No obstante, una alta presencia de esos sistemas en la red puede fotovoltaicos puede resultar en problemas de sobretensión [2]. Otras grandezas eléctricas que son modificadas debido a la presencia de los sistemas fotovoltaicos son las pérdidas en la red [3] y la carga del transformador [4].

Durante el análisis del comportamiento de la red eléctrica ante la presencia de los sistemas fotovoltaicos, algunos autores especificaron previamente la localización o la capacidad de esos sistemas [5], [6]. Esto puede ser así en redes de media tensión, dónde la concesionaria puede tener ese poder de decisión. No obstante, en las redes de baja tensión, la decisión para instalar los sistemas fotovoltaicos es realizada por los propietarios. Así, un enfoque que tome en cuenta las incertezas de la localización y la capacidad de los sistemas fotovoltaicos debe ser considerado en el análisis de redes de baja tensión.

\footnotetext{
* Corresponding author.:

E-mail: joelitoilha@gmail.com
} 
También, se observó que en varios trabajos fueron considerados un único o pocos niveles de penetración fotovoltaica (relación entre el número de usuarios con sistemas fotovoltaicos y el total de usuarios) en sus análisis [5], [7]. Sin embargo, un análisis de ese tipo es limitado para determinar cuándo la concesionaria de energía deberá tomar medidas contra problemas técnicos ocasionados por la generación fotovoltaica.

Durante la revisión literaria también fue observado que el análisis de impacto de la generación fotovoltaica en la red eléctrica fue realizado considerando solamente usuarios residenciales [8], [9]. No obstante, dado que diferentes tipos de usuarios pueden ser encontrados en las redes de baja tensión, las conclusiones alcanzadas considerando un único tipo de consumidor no pueden ser generalizadas.

Además, en muchas ocasiones, los estudios de aplicación fueron realizados asumiendo que la red de distribución está balanceada (modelo monofásico) [6], [10]. Sin embargo, las redes de distribución, especialmente las redes de baja tensión, son generalmente desbalanceadas. Así, durante el análisis de la red es trascendental llevar en cuenta esa característica desbalanceada, a fin de obtener resultados más exactos.

\section{INSERCIÓN DE LOS SISTEMAS FOTOVOLTAICOS EN LAS REDES DE BAJA TENSIÓN}

Debido a la preocupación por el calentamiento global, diversos países han firmado tratados para reducir las emisiones de $\mathrm{CO}_{2}$ [11]. Entre los acuerdos adoptados puede ser mencionado el uso de fuentes de energía renovables, como por ejemplo la energía solar. Esa fuente de energía puede ser convertida en energía eléctrica a través de los sistemas fotovoltaicos.

Desde hace varios años, países como Australia, Japón y Brasil están ofreciendo incentivos económicos a la generación fotovoltaica. Como resultado, la presencia de sistemas fotovoltaicos en redes de baja tensión está aumentado [12], [13]. Por lo tanto, es importante analizar el comportamiento de la red para varios niveles de penetración fotovoltaica.

Dado que la decisión para instalar o no los sistemas fotovoltaicos es realizada por los propietarios de las residencias, la localización de esos sistemas en la red de baja tensión es incierta.

El dimensionamiento de la capacidad de los sistemas fotovoltaicos depende entre otros factores de la demanda de energía de los usuarios. En general, es observado que las instalaciones fotovoltaicas usadas para aplicaciones residenciales, raramente superan los $10 \mathrm{~kW}$ [13]. Dado que en la red de baja tensión pueden ser encontrados usuarios con diferentes hábitos de consumo, la capacidad de los sistemas fotovoltaicos que serán instalados es incierta.

Por lo expuesto en los dos párrafos anteriores, se nota que la integración de los sistemas fotovoltaicos en la red de baja tensión presenta una naturaleza estocástica. Así, una metodología que lleve en cuenta esa naturaleza durante el análisis de la red de baja tensión es más adecuado.

\section{METODOLOGÍA}

En este trabajo es presentada una metodología para analizar el comportamiento de redes de baja tensión ante la presencia de generación fotovoltaica.

Durante el análisis, el nivel de inserción de los sistemas fotovoltaicos en la red es medido usando el concepto de penetración fotovoltaica. Diferentes definiciones de la penetración fotovoltaica han sido adoptadas en la literatura especializada [14]. En este trabajo es asumida la definición de penetración fotovoltaica, $\alpha$, indicada en (1):

$$
\alpha=\frac{u_{S F V}}{u_{T}},
$$

Donde $n_{S F V}$ es el número de usuarios con sistemas fotovoltaicos y $n_{T}$ es el número de usuarios totales conectados a la red.

Es importante notar que la consideración de diferentes niveles de penetración fotovoltaica posibilita la identificación del valor de penetración fotovoltaica para el cuál determinado problema técnico, como por ejemplo la sobretensión, aparece en la red eléctrica.

Dado que la integración fotovoltaica en la red presenta una naturaleza estocástica, en la metodología presentada, múltiples escenarios son considerados en cada nivel de penetración. Estos escenarios representan los posibles escenarios de integración de los sistemas fotovoltaicos en la red eléctrica. El número total de posibles escenarios de integración fotovoltaica en la red de baja tensión, especialmente en aquellas extensas, puede ser un número muy alto [15]. Por lo tanto, realizar el análisis de la red considerando todos los posibles escenarios no es práctico.

Para superar el inconveniente citado, en este trabajo, el método de simulación de Monte Carlo es considerado. Usando este método, un número $K$ menor que el total de posibles escenarios de integración fotovoltaica es considerado en el análisis. En este trabajo, el criterio que es adoptado para determinar el número total de escenarios que serán simulados, es el de obtener un resultado estable del valor esperado de la tensión en la red. 


\subsection{GENERACIÓN DE LOS ESCENARIOS DE INTEGRACIÓN FOTOVOLTAICA}

En la Fig. 1 es mostrado el flujograma utilizado para generar cada uno de los $K$ escenarios de integración fotovoltaica en la red eléctrica. Los datos de entrada son el número de usuarios conectados a la red de baja tensión $\left(u_{T}\right)$, el nivel de penetración fotovoltaica $(\alpha)$ y las capacidades de los sistemas fotovoltaicos normalmente utilizados en aplicaciones de baja tensión.

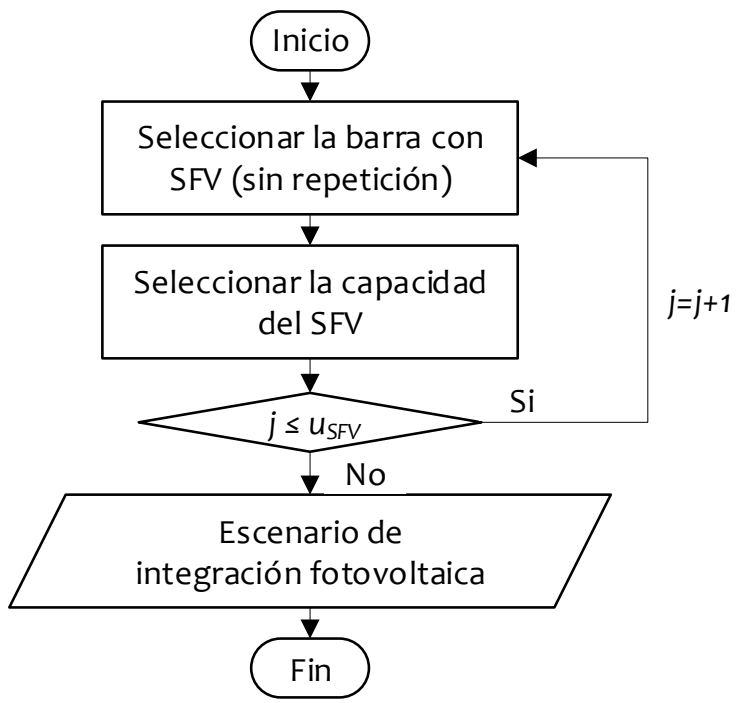

Fig. 1. Flujograma para generar los escenarios de integración fotovoltaica

El flujograma tiene 2 pasos:

Paso 1: Para cada nivel de penetración fotovoltaica $\alpha$, los $K$ escenarios de integración son generados usando el método de Monte Carlo, a través de la asociación de una probabilidad a las barras. Debido a que, por razones de privacidad, la información socioeconómica de usuarios individuales no está disponible. Es difícil determinar cuál usuario tendrá mayor probabilidad de instalar un sistema fotovoltaico. Teniendo esto en cuenta, en la metodología presentada, la misma probabilidad es asignada a todas las barras. También, a fin de garantizar que cada usuario instale únicamente un sistema fotovoltaico, la selección de las barras que contendrán sistemas fotovoltaicos es realizada sin repetición.

Paso 2: La potencia del sistema fotovoltaico es seleccionada aleatoriamente entre las potencias normalmente usadas en aplicaciones de baja tensión. Durante la selección de las capacidades de los sistemas fotovoltaicos es considerado que los usuarios no pueden instalar sistemas fotovoltaicos con capacidades mayores a su potencia contratada, pues tal restricción es a veces exigida por las concesionarias [16]. Si existe información de las potencias instaladas, entonces esa información puede ser utilizada para realizar una selección aleatoria ponderada de las capacidades.
Los pasos 1 y 2 se repiten tantas veces como el número de usuarios con sistemas fotovoltaicos, $u_{S F V}$, el cual es calculado usando (1).

Si en el Paso 2 del flujograma presentado, la información de las capacidades instaladas está disponible, las probabilidades asociadas a esas capacidades pueden ser calculadas como indicada en (2).

$$
p_{x}=\frac{C_{x}}{\sum C_{x}}
$$

Dónde $C_{x}$ representa el número de sistemas fotovoltaicos de capacidade $x$.

\section{ESTUDIO DE APLICACIÓN}

La metodología propuesta es aplicada en un alimentador de baja tensión trifásico desbalanceado al cual están conectados 16 usuarios residenciales (Red I). Los usuarios están conectados a la red de manera monofásica usando una fase ( $R, S$ o $T$ ) y el conductor neutro $(\mathrm{N})$, tal como se muestra en la Fig. 2.

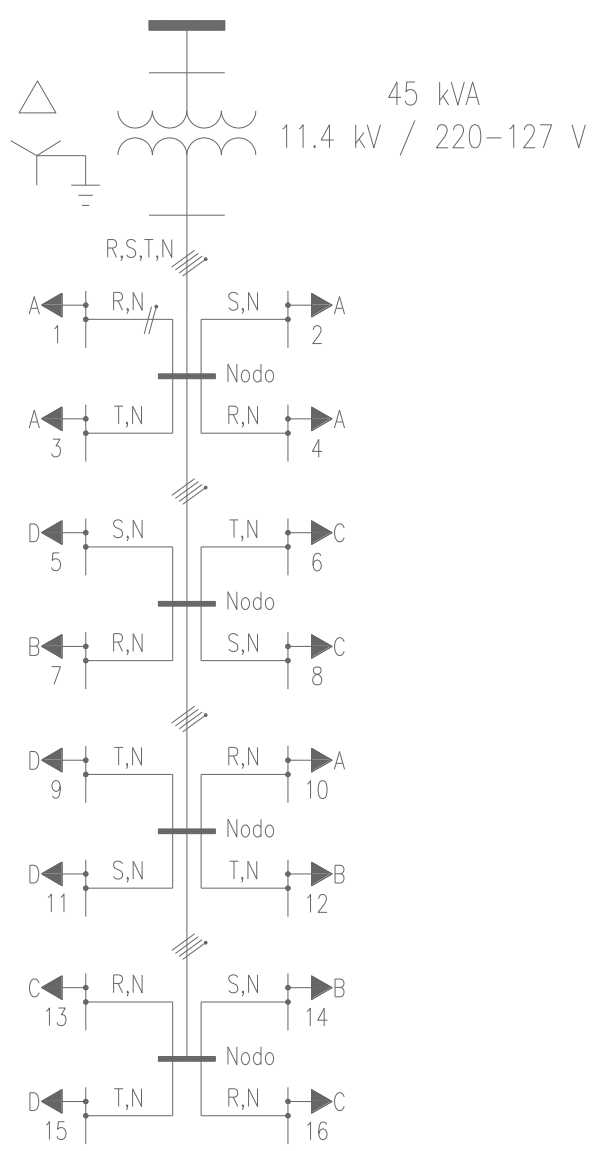

Fig. 2. Diagrama unifilar de la Red I

Cuatro clases de usuarios residenciales son considerados (A, B, C, y D), los cuales fueron clasificados en función a su consumo de energía mensual. Durante las simulaciones es asumido un factor de potencia de 0.95 para los usuarios residenciales. 
Las informaciones de los sistemas fotovoltaicos instalados en la ciudad dónde se encuentra la red en análisis indican que las capacidades de esos sistemas están entre 2 kW a 6 kW [13]. La frecuencia con la cuál las diferentes potencias fueron instaladas son usadas durante la generación de escenarios de integración fotovoltaica.

Con la finalidad de identificar como las grandezas eléctricas de la red (v.g., la tensión en las barras y las pérdidas activas en las líneas) cambian con la presencia de usuarios comerciales, dos usuarios residenciales de la Red I son substituidos por usuarios comerciales. La conexión de esos usuarios a la red es trifásica. Los usuarios sustituidos fueron los usuarios 8 y 15. La potencia de los sistemas fotovoltaicos considerada en esas barras fue de $10 \mathrm{~kW}$. La red así modificada es denominada como Red II en este trabajo. Para los usuarios comerciales, un factor de potencia igual a 0.92 es asumido.

Los perfiles de demanda de los usuarios residenciales y comerciales, así como el perfil de irradiancia solar (todos en p.u.) son mostrados en la Figura 3. Para obtener ese perfil fueron considerados los respectivos valores máximos como los valores base.

Es observado en la Fig. 3 que la curva de irradiancia solar (en consecuencia, la generación fotovoltaica) no presenta buena coincidencia con los perfiles de demanda residencial. Esto porque los valores de alta irradiancia solar ocurren en periodos diferentes a los valores pico de demanda. De manera diferente, es observado que el perfil de demanda de los usuarios comerciales sí presenta buena coincidencia con la curva de irradiancia solar.

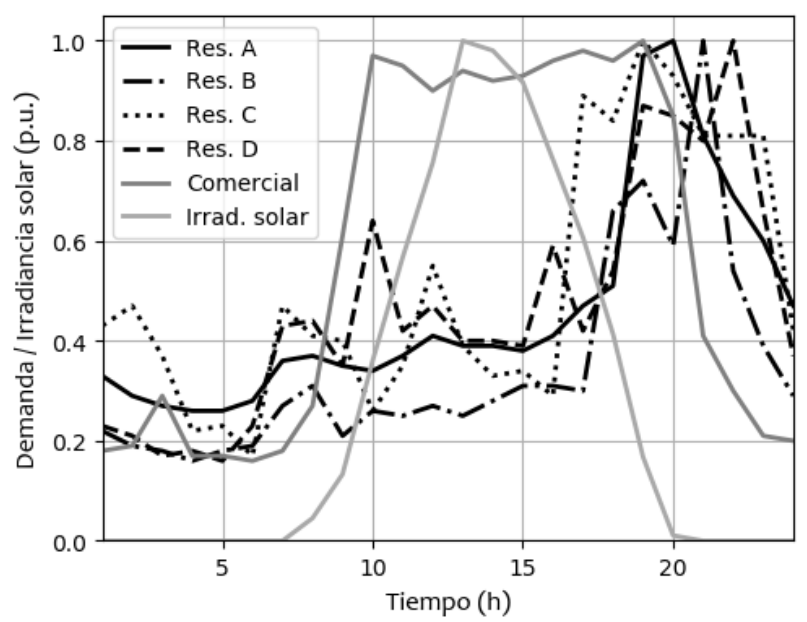

Fig. 3. Perfiles de demanda de los usuarios (p.u.) y perfil de irradiancia solar(p.u.)

Para fines de análisis del comportamiento de la red de baja tensión ante la presencia de la generación fotovoltaica, las Redes I y II descritas anteriormente son analizada en dos casos: el Caso base y el Caso SFV. En el Caso base, la red es analizada sin la presencia de los sistemas fotovoltaicos. En el Caso SFV, la red es analizada considerando diferentes niveles de penetración fotovoltaica y diferentes escenarios de integración fotovoltaica, los cuales son generados usando la metodología presentada en la Sección 3. El número de usuarios con sistemas fotovoltaicos es incrementado de uno en uno hasta alcanzar una penetración fotovoltaica de $50 \%\left(\alpha_{i}=50 \%\right)$. Usando (1), los niveles de penetración fotovoltaica equivalen aproximadamente a $6 \%, 12 \%, 19 \%, 25 \%, 31 \%, 38 \%, 44 \%$, y $50 \%$.

Después de un análisis, fue concluido que la consideración de $K=200$ escenarios de integración fotovoltaica en cada nivel de penetración fotovoltaica resulta en valores estables del valor esperado de la tensión. Así, ese número de escenarios fue considerado durante las simulaciones de flujos de potencia de cada uno de los niveles de penetración fotovoltaica considerados en el Caso SFV.

Los flujos de potencia son simulados utilizando el OpenDSS [17], el cual es Ilamado externamente usando Python [18]. A fin de tomar en cuenta la variabilidad de la generación fotovoltaica y la demanda, las simulaciones son realizadas a lo largo de un día.

\subsection{TENSIÓN EN LA RED}

En las Tablas I y II es indicado para los niveles de penetración fotovoltaica que resultaron en barras con problemas de sobretensión, el número máximo de barras de barras con sobretensión (NBS), las barras que presentan sobretensión y la tensión en las respectivas barras. Las Tablas I y II fueron elaboradas considerando el escenario que resultó con el mayor número de barras con sobretensión y para el cual el aumento de tensión en las barras fue mayor (peor escenario).

TABLA I

Sobretensión en las barras considerando el peor escenario - Red I

\begin{tabular}{|c|c|c|c|}
\hline$\alpha_{i}$ & NBS & Barras con sobretensión & Tensión (p.u.) \\
\hline $25 \%$ & 2 & {$\left[\begin{array}{lll}13 & 16\end{array}\right]$} & {$\left[\begin{array}{lll}1.053 & 1.053]\end{array}\right.$} \\
\hline $31 \%$ & 2 & {$[1316]$} & {$[1.0531 .053]$} \\
\hline $38 \%$ & 3 & {$\left[\begin{array}{lll}9 & 12 & 15\end{array}\right]$} & {$\left[\begin{array}{llll}1.056 & 1.055 & 1.058\end{array}\right]$} \\
\hline $44 \%$ & 4 & {$\left[\begin{array}{llll}7 & 10 & 13 & 16\end{array}\right]$} & {$\left[\begin{array}{llll}1.051 & 1.055 & 1.057 & 1.054\end{array}\right]$} \\
\hline $50 \%$ & 5 & {$\left[\begin{array}{lllllll}9 & 12 & 13 & 15 & 16\end{array}\right]$} & {$\left[\begin{array}{lllll}1.055 & 1.055 & 1.052 & 1.058 & 1.052\end{array}\right]$} \\
\hline
\end{tabular}

TABLA II

Sobretensión en las barras considerando el peor escenario - Red II

\begin{tabular}{|c|c|c|c|}
\hline$\alpha_{i}$ & NBS & Barras con sobretensión & Tensión (p.u.) \\
\hline $44 \%$ & 4 & {$\left[\begin{array}{llll}7 & 10 & 13 & 16\end{array}\right]$} & {$\left[\begin{array}{llllll}1.05 & 1.053 & 1.054 & 1.051\end{array}\right]$} \\
\hline $50 \%$ & 5 & {$\left[\begin{array}{lllllllll}7 & 10 & 13 & 15 & 16\end{array}\right]$} & {$\left[\begin{array}{lllll}1.051 & 1.054 & 1.055 & 1.051 & 1.052\end{array}\right]$} \\
\hline
\end{tabular}


Se nota que tanto en la Red I como en la Red II, el número de barras con sobretensión presenta relación directa con la penetración fotovoltaica. El problema de sobretensión aparece primero en la Red I para $\alpha_{i}=$ $25 \%$ y luego en la Red II para $\alpha_{i}=44 \%$. Se nota también que, para el mismo nivel de penetración, los valores de tensión en las barras con sobretensión de la Red I son mayores de aquellas encontradas en la Red II. Por lo tanto, la presencia de cargas comerciales en la Red II, mitiga el aumento de tensión ocasionado por la generación fotovoltaica.

En la Red I, el problema de sobretensión tiene lugar primero en las barras alejadas del transformador. Por ejemplo, para $\alpha_{i}=25 \%$, las barras 13 y 16 ya presentan problemas de sobretensión. De manera contraria, las barras más próximas del transformador (v. g., barra 7) sólo presentan problemas de sobretensión para valores de penetración fotovoltaica relativamente altos, tal como $\alpha_{i}=44 \%$.

Es importante recordar al lector que los resultados indicados en las Tablas I y II corresponden al peor escenario. Por lo tanto, en otros escenarios, el número de barras con sobretensión puede ser menor o igual a los indicados en las Tablas I y II. Así mismo, las barras con problemas de sobretensión pueden ser diferentes a las indicadas en las Tablas I y II, no obstante, con menores valores de tensión. Por ejemplo, la distribución de frecuencias de la sobretensión en las barras de la Redes I y II, considerando $\alpha_{i}=50 \%$, son mostradas en las Figuras 4 y 5 , respectivamente.

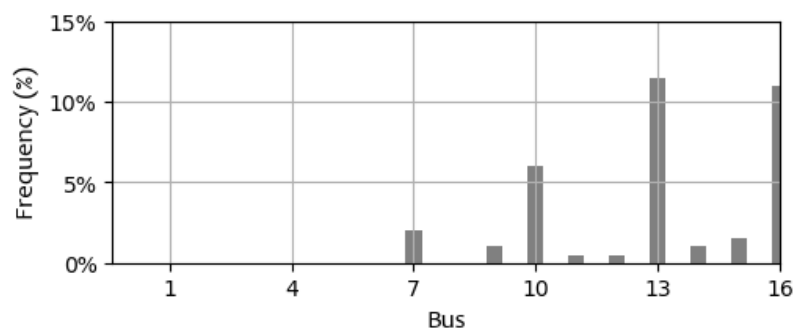

Fig. 4. Distribución de frecuencias de la sobretensión en las barras, $\alpha_{i}=50 \%$ - Red I

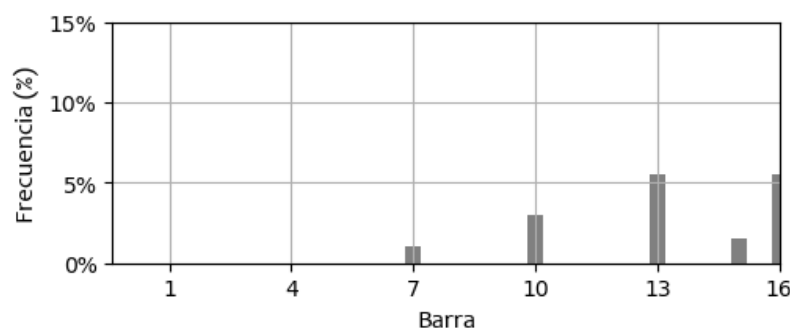

Fig. 5. Distribución de frecuencias de la sobretensión en las barras, $\alpha_{i}=50 \%$ - Red II

Se observa que barras diferentes a las indicadas en la Tabla I también presentan el problema de sobretensión. También, tanto en la Red I como en la Red II, las barras más alejadas del transformador son más propensas a presentar el problema de sobretensión. Entre las barras con similar alejamiento del transformador (v. g., barras 13, 14, 15 y 16), la propensión a la sobretensión es más pronunciada en las barras conectadas a la fase C (barras 13 y 16). Por lo tanto, es importante considerar el modelo trifásico de la red durante la evaluación de su comportamiento ante la presencia de la generación fotovoltaica.

\subsection{PÉRDIDAS ACTIVAS EN LAS LÍNEAS}

A partir de la Tabla I es observado que el problema de sobretensión aparece en la Red I a partir de $\alpha_{i}=$ $25 \%$. Teniendo en cuenta esto y debido a que, en el presente trabajo, con excepción del ajuste del tap, no es considerado otro mecanismo de regulación de tensión, a seguir, la Red I y Red II son analizadas en mayor detalle para $\alpha_{i}=19 \%$.

En las Fig. 6 y 7 son mostrados las pérdidas de potencia activa en la Red I y Red II, respectivamente.

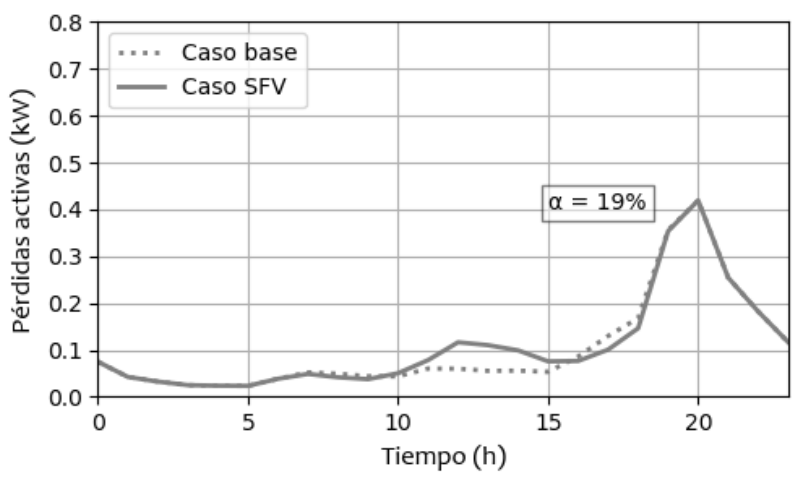

Fig. 6. Pérdidas activas en las líneas - Red I

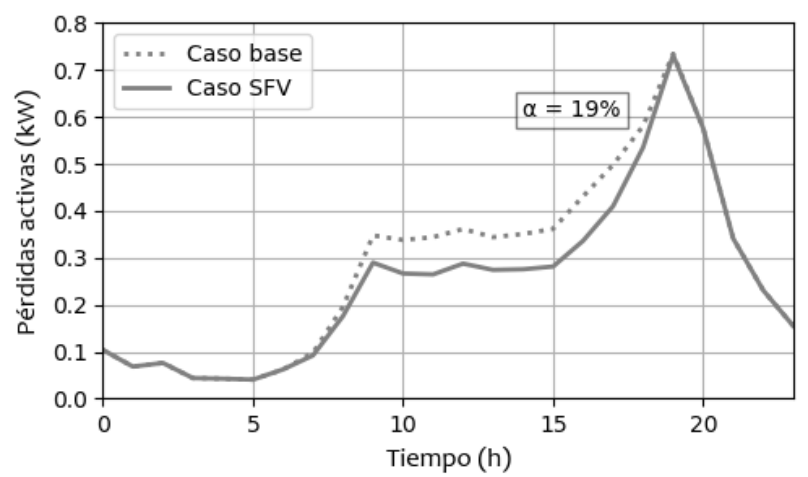

Fig. 7. Pérdidas activas en las líneas - Red II

Para la Red I es observado que la presencia de los sistemas fotovoltaicos resulta en un incremento de las pérdidas activas del Caso base, sobre todo en las horas de mayor irradiancia solar. De manera opuesta, en la Red II, la presencia de los sistemas fotovoltaicos resulta en una reducción de las pérdidas activas del Caso base. Por lo tanto, el desempeño de la red eléctrica es mejorado cuando el nivel de penetración fotovoltaica es 
bajo y existen usuarios cuyas demandas presentan buena coincidencia con la generación fotovoltaica (Red II).

Para el Caso base, las pérdidas activas diarias en las Redes I y II son $2.45 \mathrm{kWh}$ y $6.73 \mathrm{kWh}$, respectivamente. El valor esperado de las pérdidas diarias en el Caso SFV (como porcentaje de los valores encontrados en el Caso base) son indicados en la Tabla III.

TABLA III

Valor esperado de las pérdidas activas diarias en las líneas en el Caso SFV como porcentaje del valor encontrado en el Caso base

\begin{tabular}{l|cccccccc}
\hline \hline \multirow{2}{*}{ Red } & \multicolumn{7}{|c}{ Penetración fotovoltaica $\left(\alpha_{i}\right)$} \\
& $6 \%$ & $12 \%$ & $19 \%$ & $25 \%$ & $31 \%$ & $38 \%$ & $44 \%$ & $50 \%$ \\
\hline I & $101 \%$ & $103 \%$ & $105 \%$ & $110 \%$ & $116 \%$ & $120 \%$ & $126 \%$ & $133 \%$ \\
II & $96 \%$ & $93 \%$ & $89 \%$ & $86 \%$ & $84 \%$ & $82 \%$ & $80 \%$ & $79 \%$ \\
\hline \hline
\end{tabular}

Se nota que, en la Red I, las pérdidas se incrementan con la penetración fotovoltaica. Las pérdidas aumentan incluso desde bajos niveles de penetración fotovoltaica como $\alpha_{i}=6 \%$.

Para la Red II, las pérdidas disminuyen con la penetración fotovoltaica, incluso para valores de penetración fotovoltaica relativamente altos como $\alpha_{i}=50 \%$. No obstante, simulaciones adicionales, realizadas para niveles de penetración mayores a los indicados en la Tabla III, indican que a partir de $\alpha_{i}=$ $69 \%$, las pérdidas comienzan a crecer.

\section{CONCLUSIONES}

- Una metodología para analizar el comportamiento de redes de baja tensión ante la presencia de generación fotovoltaica fue presentada. El método considera la naturaleza estocástica de la integración fotovoltaica en la red a través de escenarios. Diferentes niveles de penetración fotovoltaica fueron considerados durante el análisis. En el estudio de caso es considerado una red con consumidores residenciales (Red I) y otra con consumidores residenciales $y$ comerciales (Red II). Para llevar en cuenta la variabilidad de la demanda y la generación fotovoltaica, las simulaciones son realizadas a lo largo de un día. A partir de los resultados fueron alcanzadas las siguientes conclusiones.

- El problema de sobretensión presenta relación directa con la penetración fotovoltaica. Las barras más alejadas del transformador son más propensas a presentar problemas de sobretensión. La propensión a la sobretensión es influenciada por la naturaleza desbalanceada de la red de baja tensión. La consideración de varios niveles de penetración fotovoltaica y múltiples escenarios permite encontrar el nivel de penetración fotovoltaica para el cual el problema de sobretensión aparece en la red eléctrica.

- Cuando las curvas de demanda y generación fotovoltaica no presentan buena coincidencia (Red I), las pérdidas activas en la red pueden aumentar incluso para bajos niveles de penetración fotovoltaica.

- Cuando las curvas de demanda y generación fotovoltaica presentan buena coincidencia (Red II), las pérdidas activas en la red disminuyen para penetraciones fotovoltaicas moderadas. Sin embargo, las pérdidas comienzan a aumentar para altos niveles de penetración fotovoltaica. Por lo tanto, las pérdidas activas en la red dependen de las condiciones de demanda y generación fotovoltaica

\section{AGRADECIMIENTOS}

Este estudio fue financiado en parte por la Coordenação de Aperfeiçoamento de Pessoal de Nível Superior - Brasil (CAPES) - Código de financiamiento 001, por la Fundação de Amparo à Pesquisa do Estado de São Paulo (FAPESP) bajo concesiones 2015/21972-6 y 2017/22577-9, y por la CNPq bajo concesiones 307281/2016-7 and 422044/2018-0.

\section{REFERENCIAS}

[1] IEA Photovoltaic Power System Programme (IEA PVPS. 2019 Snapshot of Global PV Markets , 2019

[2] R. Torquato, D. Salles, C. Oriente Pereira, P. C. M. Meira y W. Freitas, "A Comprehensive Assessment of PV Hosting Capacity on Low-Voltage Distribution Systems," IEEE Trans. Power Deliv., vol. 33, no. 2, pp. 1002-1012, Apr. 2018.

[3] R. S. C. Camargos, R. A. Shayani y M. A. G. de Oliveira, "Evaluation whether photovoltaic distributed generation postpones or anticipates reinforcements detected by distribution network expansion planning," IET Gener. Transm. Distrib., vol. 13, no. 7, pp. 1036-1048, Apr. 2019.

[4] H. Pezeshki, P. J. Wolfs y G. Ledwich, "Impact of High PV Penetration on Distribution Transformer Insulation Life," IEEE Trans. Power Deliv., vol. 29, no. 3, pp. 1212-1220, Jun. 2014.

[5] S. Pukhrem, M. Basu, M. F. Conlon, and K. Sunderland, "Enhanced Network Voltage Management Techniques Under the Proliferation of Rooftop Solar PV Installation in Low-Voltage Distribution Network," IEEE J. Emerg. Sel. Top. Power Electron., vol. 5, no. 2, pp. 681-694, Jun. 2017.

[6] M. Ahmadi, M. E. Lotfy, R. Shigenobu, A. Yona y T. Senjyu, "Optimal sizing and placement of rooftop solar photovoltaic at Kabul city real distribution network," IET Gener. Transm. Distrib., vol. 12, no. 2, pp. 303-309, Jan. 2018.

[7] Y. Xu, Z. Y. Dong, R. Zhang y D. J. Hill, “Multi-Timescale Coordinated Voltage/Var Control of High Renewable-Penetrated Distribution Systems," IEEE Trans. Power Syst., vol. 32, no. 6, pp. 4398-4408, Nov. 2017.

[8] D. Schwanz, F. Moller, S. K. Ronnberg, J. Meyer y M. H. J. Bollen, "Stochastic Assessment of Voltage Unbalance Due to Single-PhaseRevista TECNIA Vol.31 N² Enero-Junio 2021 
Connected Solar Power," IEEE Trans. Power Deliv., vol. 32, no. 2, pp 852-861, Apr. 2017.

[9] A. Navarro-Espinosa y L. F. Ochoa, "Probabilistic Impact Assessment of Low Carbon Technologies in LV Distribution Systems," IEEE Trans. Power Syst., vol. 31, no. 3, pp. 2192-2203, 2016.

[10] C. Zhang, Y. Xu, Z. Dong y J. Ravishankar, “Three-Stage Robust Inverter-Based Voltage/Var Control for Distribution Networks with High-Level PV," IEEE Trans. Smart Grid, vol. 10, no. 1, pp. 782-793, 2019. [11] REN21, "Renewables 2019 Global Status Report," Paris, 2019. [12] B. Mountain and P. Szuster, "Solar Everywhere," IEEE power energy Mag., no. August, pp. 53-60, 2015.

[13] Agência Nacional de Energia Elétrica - ANEEL. Geração distribuída 2019. [En linea]. Disponible en: http://www2.aneel.gov.br/scg/gd/GD_Estadual.asp.

[14] T. Aziz y N. Ketjoy, "PV Penetration Limits in Low Voltage
Networks and Voltage Variations," IEEE Access, vol. 5, pp. 1678416792, 2017.

[15] A. Dubey y S. Santoso, "On Estimation and Sensitivity Analysis of Distribution Circuit's Photovoltaic Hosting Capacity," IEEE Trans. Power Syst., vol. 32, no. 4, pp. 2779-2789, 2017.

[16] Agência Nacional de Energia Elétrica - ANEEL.(17, abr 2012). Resolução normativa $N^{\circ} 482$, Digital Times [En línea]. Disponible en: http://www2.aneel.gov.br/cedoc/bren2012482.pdf.

[17] Electric Power Research Institute - EPRI. (2018). OpenDSS Manual," 2018. [En linea]. Disponible en: https://smartgrid.epri.com/SimulationTool.aspx

[18] Python Software Foundation. (2019). Python Language Reference [En linea]. Disponible en: https://docs.python.org/3/reference/. 